CORRIGENDUM

doi:10.1038/nature10221

\title{
CD95 promotes tumour growth
}

Lina Chen, Sun-Mi Park, Alexei V. Tumanov, Annika Hau,

Kenjiro Sawada, Christine Feig, Jerrold R. Turner, Yang-Xin Fu,

Iris L. Romero, Ernst Lengyel \& Marcus E. Peter

Nature 465, 492-496 (2010)

In our recent Corrigendum (Nature 471, 254 (2011); doi:10.1038/ nature09897), Fig. 4g inadvertently contained three incorrect panels. The corrected Fig. $4 \mathrm{~g}$ is shown below. This mistake does not alter the overall conclusions of this Letter.

g

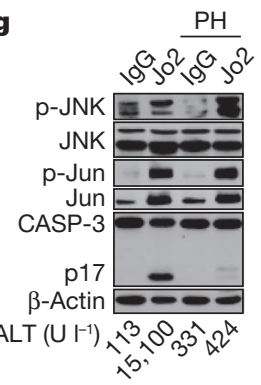

